\title{
Study of antioxidant activity of fullerenols by inhibition of adrenaline autoxidation
}

\author{
I. A. Dubinina ${ }^{1,2}$, E. M. Kuzmina ${ }^{1}$, A. I. Dudnik ${ }^{2}$, N. G. Vnukova ${ }^{1,2}$, \\ G. N. Churilov ${ }^{1,2}$, N. A. Samoylova ${ }^{3}$ \\ ${ }^{1}$ Siberian Federal University, Krasnoyarsk, Russia \\ ${ }^{2}$ Kirensky Institute of Physics SB RAS, Krasnoyarsk, Russia \\ ${ }^{3}$ Leibniz Institute for Solid State and Materials Research, Dresden, Germany \\ churilov@iph.krasn.ru
}

PACS 81.05.ub

DOI 10.17586/2220-8054-2016-7-1-153-157

In this paper, we describe application of the adrenaline autoxidation reaction to determine the antioxidant activity of fullerenols $\mathrm{C}_{60}, \mathrm{C}_{70}$ their mixture with higher fullerenol and endohedral fullerenol $\mathrm{Y} @ \mathrm{C}_{82}$. It was shown that the adrenaline autoxidation reaction can be applied to determine the antioxidant activity of fullerenols. The antioxidant activity of $\mathrm{C}_{70}$ fullerenol was higher than that of $\mathrm{C}_{60}$ fullerenol Additionally, the antioxidant activity of $\mathrm{Y} @ \mathrm{C}_{82}$ fullerenol was higher than that of $\mathrm{C}_{70}$ fullerenol.

Keywords: fullerenol, antioxidant activity, endohedral fullerenol, adrenaline autoxidation.

Received: 20 November 2015

\section{Introduction}

Polyhydroxylated fullerenes have been developed as the main derivative of fullerenes with excellent biocompatibility and biofunctionality [1,2]. Indeed, $\mathrm{C}_{60}$ fullerenols have been found to have outstanding antioxidant activity, which scavenge almost all reactive oxygen species (ROS) and reactive nitrogen species (RNS) [3].

Endohedral metallofullerenes, i.e. molecules in which a fullerene encapsulates a metal atom(s), have shown great promise for use in biomedical science. Although $\mathrm{C}_{60}$ has been the most commonly studied fullerene in biological systems, a few endohedral materials have been synthesized using $\mathrm{C}_{60}$ as a cage molecule despite the limited interior volume of $\mathrm{C}_{60}$. Therefore, most endohedral metallofullerenes are synthesized using $\mathrm{C}_{82}$ or higher molecular weight fullerenes (presumably with larger interior volumes). Our group has prepared many $\mathrm{C}_{82}$ fullerene derivatives. $\mathrm{Y} @ \mathrm{C}_{82}$ is one of the most important molecules in the metallofullerene family [4]. Yttrium endohedral metallofullerenol (e.g., Y@ $\left.\mathrm{C}_{82}(\mathrm{OH})_{22}\right)$ is a functionalized fullerene with yttrium trapped inside the $\mathrm{C}_{82}$ fullerene cage. It was reported that the chemical and physical properties of endohedral metallofullerenols depend on the number and position of the hydroxyl groups on the fullerene cage [4]. These results demonstrated that modifying the outer cage of $\mathrm{Y} @ \mathrm{C}_{82}$ with a number of hydroxyl groups tunes the electronic properties of the inner metal atom as well as the electron density and polarizability of the electrons localized on the fullerene's cage.

A number of fullerenes, fullerenols, and endohedral metallofullerenols have been shown to be capable of scavenging free radicals [3,5]. However, much less is known about the antioxidant activity of $\mathrm{Y} @ \mathrm{C}_{82}(\mathrm{OH})_{22}$. It has not been determined if the ROS-scavenging capability of $\mathrm{Y} @ \mathrm{C}_{82}(\mathrm{OH})_{22}$ is higher than that of other functionalized fullerenes. In this work, we applied 
the adrenaline autoxidation reaction to determine the antioxidant activity of fullerenols $\mathrm{C}_{60}$ and their mixture with higher and endohedral fullerenols.

\section{Methods}

\subsection{Preparation of fullerenols}

Fullerene mixture with endohedral Y-fullerene was synthesized at the set up with high frequency (HF) arc plasma for sputter graphitic electrodes (graphitic rods) [6,7]. Graphitic rods, with an axial hole in which $\mathrm{Y}_{2} \mathrm{O}_{3}$ was inserted, were annealed at $1800{ }^{\circ} \mathrm{C}$ for $20 \mathrm{~min}$. Arc current was $190 \mathrm{~A}$, frequency - $66 \mathrm{kHz}$, and chamber pressure was $98 \mathrm{kPa}$. Extraction was carried out using $\mathrm{CS}_{2}$.

Synthesized fullerenes were separated by liquid chromatography with turbostratic graphite (graphite with an interplanar distance $3.42 \AA$ ) as stationary phase and toluene/hexane (4:6) mixture as mobile phase (or pure toluene in case of $\mathrm{C}_{70}$ elution). Endohedral Y-containing fullerene was separated using HPLC chromatography (Agilent 1200-series with Buckyprep column).

The experimental preparation of fullerenols was achieved under acidic conditions by treatment with nitric acid.

\subsection{Antioxidant activity study}

Antioxidant activity (AA) of fullerenols was measured according to Hasanova et. al [8] by the inhibition of adrenaline autoxidation. The reaction mixture contained $0.1 \mathrm{~cm}^{3}$ of $0.1 \%$ adrenaline hydrochloride, $4 \mathrm{~cm}^{3}$ carbonate buffer, $\mathrm{pH} 10.55$, and $0.1 \mathrm{~cm}^{3}$ of fullerenol solution. The increase in absorbance due to adrenaline autoxidation was monitored at $347 \mathrm{~nm}$ and the percentage inhibition of the maximal rate of increase in absorbance was determined. According to [8] more than $10 \%$ inhibition rate shows the AA of investigated solution. This method has been developed for measuring AA of vegetative gathering and for the first time applied for fullerenols. The AA was calculated as follows:

$$
\mathrm{AA}=\frac{(\mathrm{D} 1-\mathrm{D} 2) \cdot 100}{\mathrm{D} 1}, \%
$$

where D1 is optical density of pure adrenaline and D2 is optical density of adrenaline with fullerenol.

\section{Results}

Synthesized Y-containing fullerene mixture was investigated by HPLC (Fig. 1). We collected fraction with Y-containing endofullerene $(30-40 \mathrm{~min})$ and investigated it by massspectrometry (Bruker BIFLEXTM III Time-of-Flight mass spectrometer with laser desorption) (Fig. 2). The fullerene mixture was shown to consist of only one type of endohedral metallofullerene- $\mathrm{Y} @ \mathrm{C}_{82}$. The fullerene mixture was separated and 3 fractions were received - pure $\mathrm{C}_{60}(99 \%), \mathrm{C}_{70}$ with higher fullerenes (92 and $8 \%$, respectively), and higher fullerenes with $6 \%$ of $\mathrm{Y} @ \mathrm{C}_{82}$.

Fullerene fractions were hydroxylated and solutions with concentrations $2 \mathrm{mg} / \mathrm{ml}$ of fullerenols were produced.

Figure 3 shows the dynamics for the adrenaline autoxidation reaction. The decrease in the optical density showed the inhibitory effect of the fullerenols. To rate the influence of fullerenols on adrenaline autoxidation process, different exposure times were chosen $-30 \mathrm{~s}$, $3 \mathrm{~min}, 5 \mathrm{~min}$. Calculation of AA showed that all fullerenols displayed great AA. The AA significantly increased with increasing concentration of higher fullerenols (Fig. 4). 


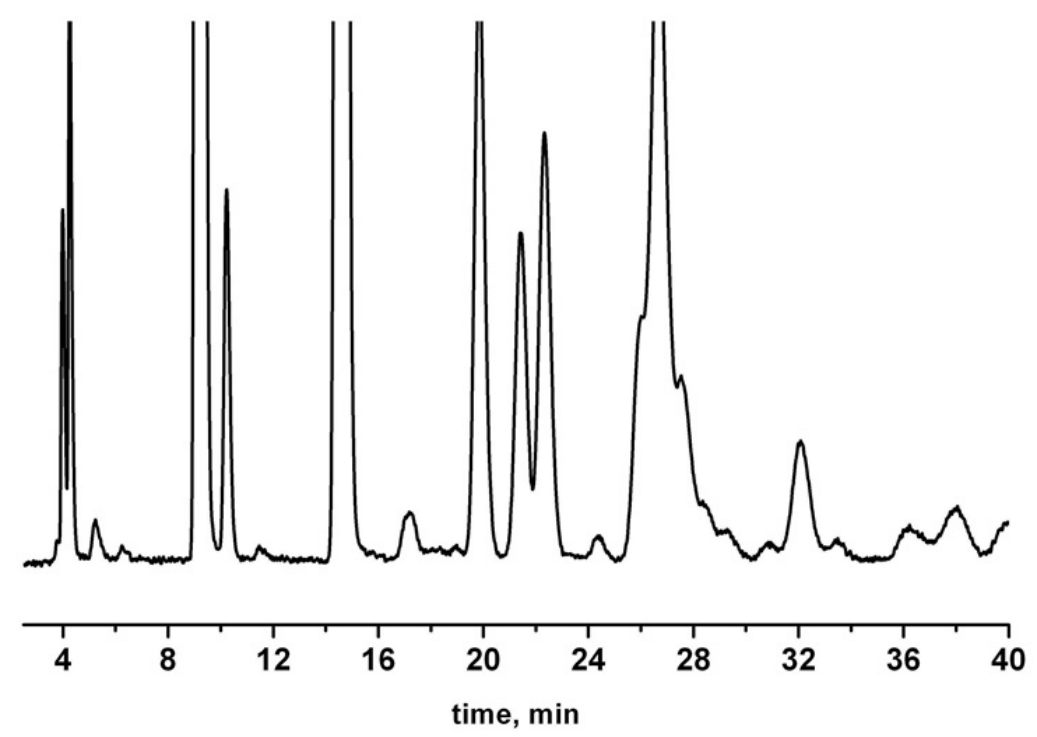

FIG. 1. Chromatogram of fullerene mixture with Y-endofullerene

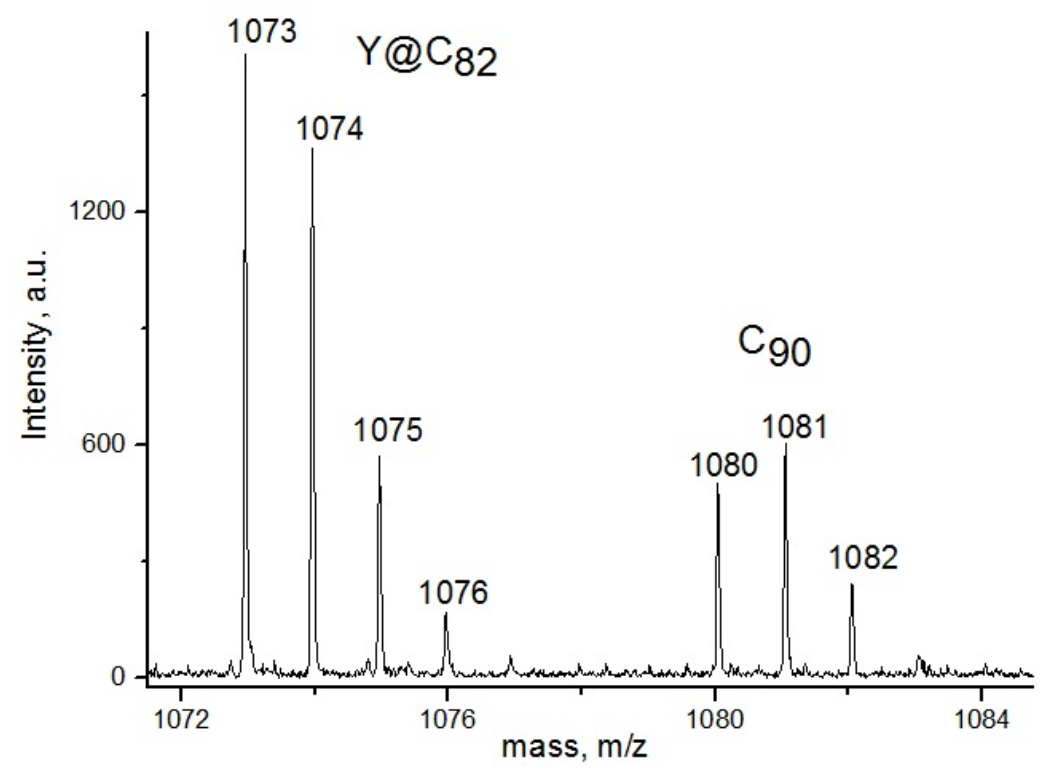

FIG. 2. Mass-spectrum of fullerene fraction with Y-endofullerene $(30-40 \mathrm{~min})$

\section{Conclusion}

Fullerenols were investigated to see if they inhibit the adrenaline autoxidation reaction and thus show great antioxidant activity. Increasing the higher fullerenol content, as well as $\mathrm{Y} @ \mathrm{C}_{82}$ content, resulted in an increase in the antioxidant activity of fullerenol solution.

\section{Acknowledgements}

The work was supported by the Russian Foundation for Basic Research 15-03-06786. 


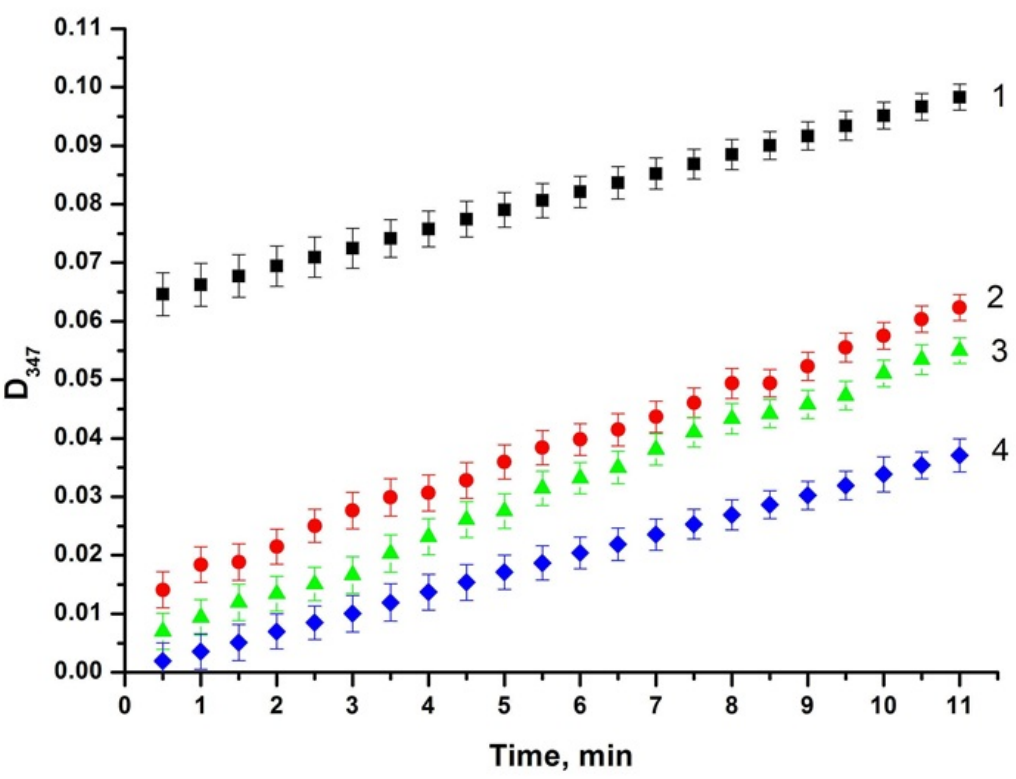

FIG. 3. Optical density dependence on reaction time. 1 - pure adrenaline, 2 $\mathrm{C}_{60}$ fullerenol, $3-\mathrm{C}_{70}$ and higher fullerenols and $4-$ endohedral and higher fullerenols

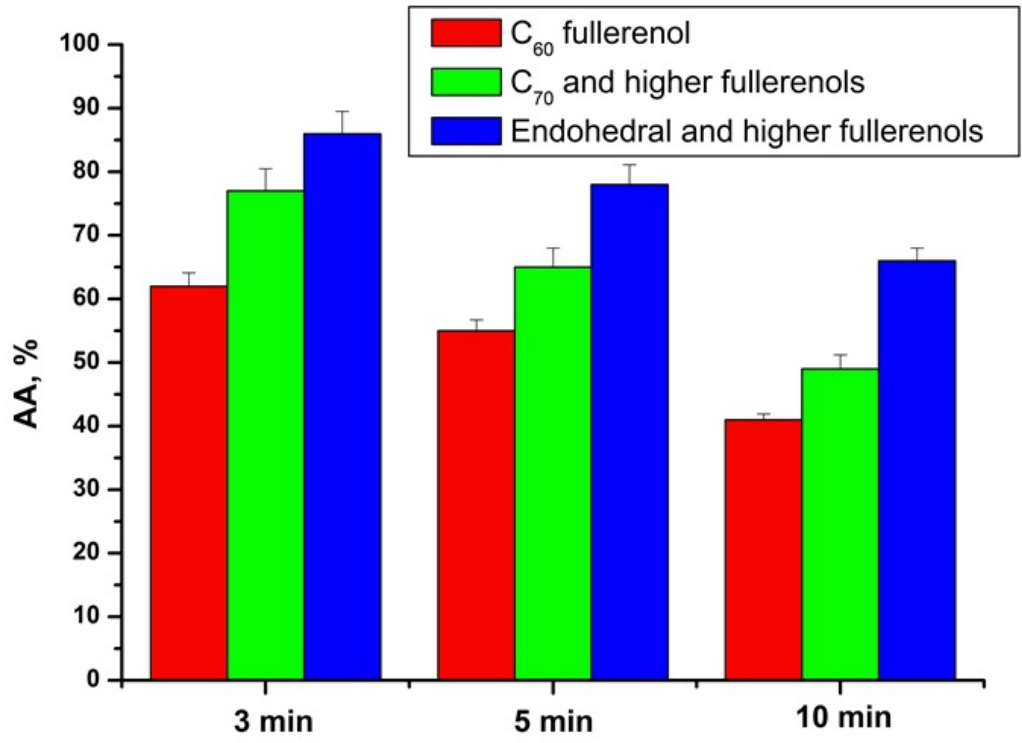

FIG. 4. Comparative antioxidant activity of fullerenols (where $100 \%$ of AA means that adrenaline autoxidation doesn't occur, and $0 \%$ means that there is no inhibitory effect). Statistically significant changes in AA level (ANOVA, $p<0.05, n=3$ ) 


\section{References}

[1] Chiang L.Y., Swirczewski J.W. Multi-hydroxy additions onto $\mathrm{C}_{60}$ fullerene molecules. J. Chem. Soc. Chem. Commun., 1992, 24, P. 1791-1793.

[2] Li J., Takeuchi A. $\mathrm{C}_{60}$ fullerol formation catalysed by quaternary ammonium hydroxides. J. Chem. Soc. Chem. Commun., 1993, 23, P. 1784-1785.

[3] Chiang L.Y., Lu F.J., Lin J.T. Free radical scavenging activity of water-soluble fullerenols. J. Chem. Soc. Chem. Commun., 1995, 12, P. 1283-1284.

[4] Tang J., Xing G.M., Zhao F., Yuan H., Zhao Y.L. Modulation of structural and electronic properties of fullerene and metallofullerenes by surface chemical modifications. J. Nanosci. Nanotechnol., 2007, 7(4-5), P. 1085-1101.

[5] Dugan L.L., Gabrielsen J.K., Yu S.P., Lin T.S., Choi D.W. Buckminsterfullerenol free radical scavengers reduce excitotoxic and apoptotic death of cultured cortical neurons. Neurobiol. Dis., 1996, 3, P. 129-135.

[6] Churilov G.N. Plasma Synthesis of Fullerenes. Instruments and Experimental Techniques, 2000, 43(1), P. 110.

[7] Churilov G.N., Kratschmer W., Osipova I.V., Glushenko G.A., Vnukova N.G., Kolonenko A.L., Dudnik A.I. Synthesis of fullerenes in a high-frequency arc plasma under elevated helium pressure. Carbon, 2013, 62, P. 389-392.

[8] Hasanova S.R., Plehanova T.I.,Gashimova D.T.,Galiahmetova E.H., Klysh E.A. Comparative study of antioxidant activity of vegetative gathering. Proc. Voronezh State University. Series: Chemistry, Biology, Pharmacy, 2007, 1, P. 163. 